\title{
Some New Delay Integral Inequalities Based on Modified Riemann-Liouville Fractional Derivative and Their Applications
}

\author{
Zhimin Zhao, Run Xu \\ Department of Mathematics, Qufu Normal University, Qufu, China \\ Email: 782493982@qq.com, xurun2005@163.com
}

Received 21 January 2015; accepted 5 May 2015; published 11 May 2015

Copyright (C) 2015 by authors and Scientific Research Publishing Inc.

This work is licensed under the Creative Commons Attribution International License (CC BY). http://creativecommons.org/licenses/by/4.0/

c) (i) Open Access

\begin{abstract}
By using the properties of modified Riemann-Liouville fractional derivative, some new delay integral inequalities have been studied. First, we offered explicit bounds for the unknown functions, then we applied the results to the research concerning the boundness, uniqueness and continuous dependence on the initial for solutions to certain fractional differential equations.
\end{abstract}

\section{Keywords}

\section{Modified, Riemann-Liouville, Fractional Derivative, Integral Inequalities, Delay Fractional} Differential Equation

\section{Introduction}

The common differential and integral inequalities are playing an important role in the qualitative analysis of differential equations. At the same time, delay integral and differential inequality have been studied due to their wide applications [1]-[3]. In recent years, the fractional differential and fractional integrals are adopted in various fields of science and engineering. In addition, the fractional differential inequalities have also been studied [4]-[10]. We also need to study the delay differential equation and delay differential inequalities when dealing with certain problems. However, to the best of our knowledge, very little is known regarding this problem [11]. In this paper, we will investigate some delay integral inequalities.

In 2008, Zhiling Yuan, et al. [3] studied the following form delay integral inequality

$$
x^{p}(t) \leq a(t)+b(t) \int_{0}^{t}\left[f(s) x^{q}(\sigma(s))+g(s) x^{r}(s)+\int_{0}^{s} h(\tau) x^{m}(\tau) \mathrm{d} \tau\right] \mathrm{d} s, \quad t \in R_{+}
$$


then they offered an explicit estimate for $x(t)$, and applied this result to research the properties of solution to certain differential equations.

In 2013, Bin Zheng and Qinghua Feng [6] put forward the following form of fractional integral inequality

$$
u^{p}(t) \leq a(t)+\frac{b(t)}{\Gamma(\alpha)} \int_{0}^{t}(t-s)^{\alpha-1}\left[g(s) u^{q}(s)+\int_{0}^{s} h(\xi) u^{r}(\xi) \mathrm{d} \xi\right] \mathrm{d} s, \quad t \geq 0,
$$

and they applied the obtained results to study the properties of solution $u(t)$.

In this paper, combining (1) and (2), we will explore the following form of delay integral inequality

$$
u^{p}(t) \leq k(t)+\frac{b(t)}{\Gamma(\alpha)} \int_{0}^{t}(t-s)^{\alpha-1}\left[f(s) u^{q}(\sigma(s))+g(s) u^{r}(s)+\int_{0}^{s} h(\tau) u^{m}(\tau) \mathrm{d} \tau\right] \mathrm{d} s, \quad t \geq 0 .
$$

Now we list some Definitions and Lemmas which can be used in this paper.

Definition 1. [6] The modified Riemann-Liouville derivative of order $\alpha$ is defined by

$$
D_{t}^{\alpha} f(t)= \begin{cases}\frac{1}{\Gamma(1-\alpha)} \frac{\mathrm{d}}{\mathrm{d} t} \int_{0}^{t}(t-\xi)^{-\alpha}(f(\xi)-f(0)) \mathrm{d} \xi, & 0<\alpha<1, \\ \left(f^{(n)}(t)\right)^{(\alpha-n)}, & n \leq \alpha<n+1, \quad n \geq 1 .\end{cases}
$$

Definition 2. [6] The Riemann-Liouville fractional integral of order $\alpha$ on the interval $[0, t]$ is defined by

$$
I^{\alpha} f(t)=\frac{1}{\Gamma(\alpha)} \int_{0}^{t}(t-s)^{\alpha-1} f(s) \mathrm{d} s .
$$

Some important properties for the modified Riemann-Liouville derivative and fractional integral are listed as follows [6] (the interval concerned below is always defined by $[0, t]$ ).

$$
\begin{gathered}
\text { (1) } D_{t}^{\alpha} t^{\gamma}=\frac{\Gamma(1+\gamma)}{\Gamma(1+\gamma-\alpha)} t^{\gamma-\alpha}, \\
\text { (2) } D_{t}^{\alpha}(f(t) g(t))=g(t) D_{t}^{\alpha} f(t)+f(t) D_{t}^{\alpha} g(t), \\
\text { (3) } D_{t}^{\alpha} f[g(t)]=f_{g}^{\prime}[g(t)] D_{t}^{\alpha} g(t)=D_{g}^{\alpha} f[g(t)]\left(g^{\prime}(t)\right)^{\alpha}, \\
\text { (4) } I^{\alpha}\left(D_{t}^{\alpha} f(t)\right)=f(t)-f(0), D_{t}^{\alpha}\left(I^{\alpha} f(t)\right)=f(t), \\
\text { (5) } I^{\alpha}\left(g(t) D_{t}^{\alpha} f(t)\right)=f(t) g(t)-f(0) g(0)-I^{\alpha}\left(f(t) D_{t}^{\alpha} g(t)\right) \text {. }
\end{gathered}
$$

Lemma 1. [3] Assume that $a \geq 0, \quad p \geq q \geq 0$, and $p \neq 0$, then

$$
a^{\frac{q}{p}} \leq \frac{q}{p} K^{\frac{q-p}{p}} a+\frac{p-q}{p} K^{\frac{q}{p}} \text { for any } K>0 .
$$

Lemma 2. [6] Let $\alpha>0, a(t), b(t), u(t)$ be continuous functions defined on $t \geq 0$.

Then for $t \geq 0$,

$$
D_{t}^{\alpha} u(t) \leq a(t)+b(t) u(t)
$$

Implies

$\left.u(t) \leq u(0) \exp \left\{\int_{0}^{\frac{t^{\alpha}}{\Gamma(1+\alpha)} b} b(s \Gamma(1+\alpha))^{\frac{1}{\alpha}}\right) \mathrm{d} s\right\}+\frac{1}{\Gamma(\alpha)} \int_{0}^{t}(t-\tau)^{\alpha-1} a(\tau) \exp \left\{-\int_{\frac{\tau^{\alpha}}{\Gamma(1+\alpha)}}^{\frac{t^{\alpha}}{\Gamma(+\alpha)}} b\left((s \Gamma(1+\alpha))^{\frac{1}{\alpha}}\right) \mathrm{d} s\right\} \mathrm{d} \tau$.

\section{Main Results}

Theorem 1 Assume that $\alpha>0, u(t), k(t), a(t), f(t), g(t), h(t) \in C\left(R_{+}, R_{+}\right)$, and $k(t), a(t)$ are 
nondecreasing functions in $t \geq 0$. If $u(t)$ satisfies the following form of delay integral inequality

$$
u^{p}(t) \leq k(t)+\frac{a(t)}{\Gamma(\alpha)} \int_{0}^{t}(t-s)^{\alpha-1}\left[f(s) u^{q}(\sigma(s))+g(s) u^{r}(s)+\int_{0}^{s} h(\tau) u^{m}(\tau) \mathrm{d} \tau\right] \mathrm{d} s, \quad t \geq 0,
$$

with the initial condition

$$
\begin{gathered}
u(t)=\phi(t), \quad t \in[\beta, 0], \\
\phi(\sigma(t)) \leq k^{\frac{1}{p}}(t), \text { for } t \in R_{+} \quad \text { with } \sigma(t) \leq 0
\end{gathered}
$$

where $p \neq 0, p \geq q \geq 0, \quad p \geq r \geq 0, \quad p \geq m \geq 0, p, q, r, m$ are constants, $\sigma(t) \in C\left(R_{+}, R\right), \sigma(t) \leq t$, $-\infty<\beta=\inf \left\{\sigma(t), t \in R_{+}\right\} \leq 0$ and $\phi(t) \in C\left([\beta, 0], R_{+}\right)$, then we have

$$
\begin{aligned}
u(t) \leq & \left\{k(t) \exp \left[a(t) \int_{0}^{\frac{t^{\alpha}}{\Gamma(1+\alpha)}} G_{1}\left((s \Gamma(1+\alpha))^{\frac{1}{\alpha}}\right) \mathrm{d} s\right]\right. \\
& \left.+\frac{a(t)}{\Gamma(\alpha)} \int_{0}^{t}(t-\tau)^{\alpha-1} H_{1}(\tau) \exp \left[-a(t) \int_{\frac{\tau^{\alpha}}{\Gamma(1+\alpha)}}^{\frac{t^{\alpha}}{\Gamma(1+\alpha)}} G_{1}\left((s \Gamma(1+\alpha))^{\frac{1}{\alpha}}\right) \mathrm{d} s\right] \mathrm{d} \tau\right\}^{\frac{1}{p}}, \quad t \geq 0 .
\end{aligned}
$$

for any $K>0$, where

$$
\begin{aligned}
& H_{1}(t)=\frac{p-q}{p} K^{\frac{q}{p}} f(t)+\frac{p-r}{p} K^{\frac{r}{p}} g(t)+\int_{0}^{t} \frac{p-m}{p} K^{\frac{m}{p}} h(\tau) \mathrm{d} \tau, \\
& G_{1}(t)=\frac{q}{p} K^{\frac{q-p}{p}} f(t)+\frac{r}{p} K^{\frac{r-p}{p}} g(t)+\int_{0}^{t} \frac{m}{p} K^{\frac{m-p}{p}} h(\tau) \mathrm{d} \tau .
\end{aligned}
$$

Proof. Fix $T \geq 0$, let

$$
\begin{gathered}
z(t)=k(T)+\frac{a(T)}{\Gamma(\alpha)} \int_{0}^{t}(t-s)^{\alpha-1}\left[f(s) u^{q}(\sigma(s))+g(s) u^{r}(s)+\int_{0}^{s} h(\tau) u^{m}(\tau) \mathrm{d} \tau\right] \mathrm{d} s, \quad t \in[0, T], \\
z^{\prime}(t)=\frac{a(T)}{\Gamma(\alpha)} \frac{\mathrm{d}}{\mathrm{d} t} \int_{0}^{t}(t-s)^{\alpha-1}\left[f(s) u^{q}(\sigma(s))+g(s) u^{r}(s)+\int_{0}^{s} h(\tau) u^{m}(\tau) \mathrm{d} \tau\right] \mathrm{d} s,
\end{gathered}
$$
that

Since $\alpha>0, u(t), k(t), a(t), f(t), g(t), h(t) \in C\left(R^{+}, R^{+}\right)$, there's exist a constant $C \geq 0$, such

$$
C=\min \left\{f(s) u^{q}(\sigma(s))+g(s) u^{r}(s)+\int_{0}^{s} h(\tau) u^{m}(\tau) \mathrm{d} \tau\right\},
$$

and $\int_{0}^{t}(t-s)^{\alpha-1}\left[f(s) u^{q}(\sigma(s))+g(s) u^{r}(s)+\int_{0}^{s} h(\tau) u^{m}(\tau) \mathrm{d} \tau\right] \mathrm{d} s$ is convergence integral, so we have

$$
\begin{aligned}
z^{\prime}(t) & =\frac{a(T)}{\Gamma(\alpha)} \int_{0}^{t} \frac{\mathrm{d}}{\mathrm{d} t}(t-s)^{\alpha-1}\left[f(s) u^{q}(\sigma(s))+g(s) u^{r}(s)+\int_{0}^{s} h(\tau) u^{m}(\tau) \mathrm{d} \tau\right] \mathrm{d} s \\
& \geq \frac{a(T) C}{\Gamma(\alpha)} \int_{0}^{t} \frac{\mathrm{d}}{\mathrm{d} t}(t-s)^{\alpha-1} \mathrm{~d} s \\
& =\frac{a(T) C}{\Gamma(\alpha)} t^{\alpha-1} \\
& \geq 0 .
\end{aligned}
$$


we have $z(t)$ is a nonnegative and nondecreasing. From (4) and (7) we get

$$
u(t) \leq z^{\frac{1}{p}}(t), \quad t \in[0, T],
$$

and

$$
D_{t}^{\alpha} z(t)=a(T)\left[f(t) u^{q}(\sigma(t))+g(t) u^{r}(t)+\int_{0}^{t} h(\tau) u^{m}(\tau) \mathrm{d} \tau\right], \quad t \in[0, T] .
$$

So for $t \geq 0$ with $\sigma(t) \geq 0$, we have

$$
u(\sigma(t)) \leq z^{\frac{1}{p}}(\sigma(t)) \leq z^{\frac{1}{p}}(t), \quad t \in[0, T],
$$

for $t \geq 0$ with $\sigma(t) \leq 0$, we have

$$
u(\sigma(t))=\phi(\sigma(t)) \leq k^{\frac{1}{p}}(t) \leq k^{\frac{1}{p}}(T) \leq z^{\frac{1}{p}}(t), \quad t \in[0, T] .
$$

Combining (10) and (11), we obtain

$$
u(\sigma(t)) \leq z^{\frac{1}{p}}(t), \quad t \in[0, T]
$$

From (8), (9) and (12) we get

$$
D_{t}^{\alpha} z(t) \leq a(T)\left[f(t) z^{\frac{q}{p}}(\sigma(t))+g(t) z^{\frac{r}{p}}(t)+\int_{0}^{t} h(\tau) z^{\frac{m}{p}}(\tau) \mathrm{d} \tau\right], \quad t \in[0, T],
$$

By Lemma 1 we have

$$
\begin{aligned}
D_{t}^{\alpha} z(t) \leq & a(T)\left\{f(t)\left[\frac{q}{p} K^{\frac{q-p}{p}} z(t)+\frac{p-q}{p} K^{\frac{q}{p}}\right]+g(t)\left[\frac{r}{p} K^{\frac{r-p}{p}} z(t)+\frac{p-r}{p} K^{\frac{r}{p}}\right]\right. \\
& \left.+\int_{0}^{t} h(\tau)\left[\frac{m}{p} K^{\frac{m-p}{p}} z(\tau)+\frac{p-m}{p} K^{\frac{m}{p}}\right] \mathrm{d} \tau\right\} \\
\leq & a(T)\left[\frac{p-q}{p} K^{\frac{q}{p}} f(t)+\frac{p-r}{p} K^{\frac{r}{p}} g(t)+\int_{0}^{t} \frac{p-m}{p} K^{\frac{m}{p}} h(\tau) \mathrm{d} \tau\right] \\
& +a(T)\left[\frac{q}{p} K^{\frac{q-p}{p}} f(t)+\frac{r}{p} K^{\frac{r-p}{p}} g(t)+\int_{0}^{t} \frac{m}{p} K^{\frac{m-p}{p}} h(\tau) \mathrm{d} \tau\right] z(t) \\
= & a(T) H_{1}(t)+a(T) G_{1}(t) z(t), \quad t \in[0, T] .
\end{aligned}
$$

Since $u(t), g(t), h(t)$ are continuous and there exists a constant $M$ satisfies

$$
\left|f(t) u^{q}(\sigma(t))+g(t) u^{r}(t)+\int_{0}^{t} h(s) u^{m}(s) \mathrm{d} s\right| \leq M
$$

for $t \in[0, \varepsilon]$, where $\varepsilon>0$. Then we get

$$
\int_{0}^{t}(t-s)^{\alpha-1}\left|f(s) u^{q}(\sigma(s))+g(s) u^{r}(s)+\int_{0}^{s} h(\tau) u^{m}(\tau) \mathrm{d} \tau\right| \mathrm{d} s \leq \frac{M}{\alpha} t^{\alpha}, \quad t \in[0, \varepsilon],
$$

so we have

$$
z(0)=k(T)
$$

Using Lemma 2 to (14) we get 


$$
\begin{aligned}
z(t) \leq & k(T) \exp \left\{a(T) \int_{0}^{\frac{t^{\alpha}}{\Gamma(1+\alpha)}} G_{1}\left((s \Gamma(1+\alpha))^{\frac{1}{\alpha}}\right) \mathrm{d} s\right\} \\
& +\frac{a(T)}{\Gamma(\alpha)} \int_{0}^{t}(t-\tau)^{\alpha-1} H_{1}(\tau) \exp \left\{-a(T) \int_{\frac{\tau^{\alpha}}{\Gamma(1+\alpha)}}^{\frac{t^{\alpha}}{\Gamma(+\alpha)}} G_{1}\left((s \Gamma(1+\alpha))^{\frac{1}{\alpha}}\right) \mathrm{d} s\right\} \mathrm{d} \tau, \quad t \in[0, T] .
\end{aligned}
$$

Letting $t=T$ in (16) and considering $T>0$ is arbitary, after substituting $T$ with $t$, we get

$$
\begin{aligned}
z(t) \leq & k(t) \exp \left\{a(t) \int_{0}^{\frac{t^{\alpha}}{\Gamma(1+\alpha)}} G_{1}\left((s \Gamma(1+\alpha))^{\frac{1}{\alpha}}\right) \mathrm{d} s\right\} \\
& +\frac{a(t)}{\Gamma(\alpha)} \int_{0}^{t}(t-\tau)^{\alpha-1} H_{1}(\tau) \exp \left\{-a(t) \int_{\frac{\tau^{\alpha}}{\Gamma(1+\alpha)}}^{\frac{t^{\alpha}}{\Gamma(1+\alpha)}} G_{1} G\left((s \Gamma(1+\alpha))^{\frac{1}{\alpha}}\right) \mathrm{d} s\right\} \mathrm{d} \tau, \quad t \geq 0 .
\end{aligned}
$$

Combining (8) and (17), we get (6).

Remark 1. Assume $k(t)=0$, then the inequalities in Theorem 1 reduce to Lemma 5 in [6].

Theorem 2. Assume that $\alpha>0, u(t), k(t), a(t), f(t), g(t), h(t), \sigma(t)$ are defined as in Theorem 1. If $u(t)$ satisfies the following form of integral inequality,

$$
u^{p}(t) \leq k(t)+\frac{a(t)}{\Gamma(\alpha)} \int_{0}^{t}(t-s)^{\alpha-1}\left[f(s) u^{q}(s)+L(s, u(\sigma(s)))+\int_{0}^{s} h(\tau) u^{m}(\tau) \mathrm{d} \tau\right] \mathrm{d} s, \quad t \geq 0,
$$

where $p \geq q>0, \quad p \geq m>0, \quad p \geq 1, \quad p, q, m$ are constants and $L, M \in C\left(R_{+}^{2}, R\right)$ satisfy

$$
0 \leq L(t, x)-L(t, y) \leq M(t, y)(x-y), \quad x \geq y \geq 0,
$$

with the condition (5) in Theorem 1, then we have

$$
u(t) \leq\left\{k(t)+\frac{a(t)}{\Gamma(\alpha)} \int_{0}^{t}(t-\tau)^{\alpha-1} H_{2}(\tau) \exp \left[-\int_{\frac{\tau^{\alpha}}{\Gamma(1+\alpha)}}^{\frac{t^{\alpha}}{\Gamma(+\alpha)}} G_{2}\left((s \Gamma(1+\alpha))^{\frac{1}{\alpha}}\right) \mathrm{d} s\right] \mathrm{d} \tau\right\}^{\frac{1}{p}}, \quad t \geq 0,
$$

where

$$
\begin{gathered}
H_{2}(t)=f(t)\left(\frac{p-q}{p} K^{\frac{q}{p}}+\frac{q}{p} K^{\frac{q-p}{p}} k(t)\right)+\int_{0}^{t}\left(\frac{p-m}{p} K^{\frac{m}{p}} h(s)+\frac{m}{p} K^{\frac{m-p}{p}} h(s) k(s)\right) \mathrm{d} s \\
+L\left(t, \frac{p-1}{p} K^{\frac{1}{p}}+\frac{1}{p} K^{\frac{1-p}{p}} k(t)\right), \quad t \geq 0, \\
G_{2}(t)=\frac{q}{p} K^{\frac{q-p}{p}} f(t) a(t)+\int_{0}^{t} \frac{m}{p} K^{\frac{m-p}{p}} h(s) a(s) \mathrm{d} s+M\left(t, \frac{p-1}{p} K^{\frac{1}{p}}+\frac{1}{p} K^{\frac{1-p}{p}} k(t)\right) \frac{1}{p} K^{\frac{1-p}{p}} a(t), \quad t \geq 0 .
\end{gathered}
$$

Proof. Let

$$
z(t)=\frac{1}{\Gamma(\alpha)} \int_{0}^{t}(t-s)^{\alpha-1}\left[f(s) u^{q}(s)+L(s, u(\sigma(s)))+\int_{0}^{s} h(\tau) u^{m}(\tau) \mathrm{d} \tau\right] \mathrm{d} s, \quad t \geq 0,
$$

Since $u(t), f(t), h(t), L(t, s)$ are nonegative functions, $z(t)$ is also nonegative and nondecreasing function, in addition, there exists a constant $N$ satisfying

$$
\left|f(t) u^{q}(t)+L(t, u(\sigma(t)))+\int_{0}^{t} h(s) u^{m}(s) \mathrm{d} s\right| \leq N
$$

for $t \in[0, \varepsilon]$, where $\varepsilon>0$. Then we get 


$$
\int_{0}^{t}(t-s)^{\alpha-1}\left|f(t) u^{q}(t)+L(t, u(\sigma(t)))+\int_{0}^{t} h(s) u^{m}(s) \mathrm{d} s\right| \leq \frac{N}{\alpha} t^{\alpha}, \quad t \in[0, \varepsilon],
$$

so we can get $z(0)=0$. From (18) we have

$$
u(t) \leq(k(t)+a(t) z(t))^{\frac{1}{p}}, \quad t \geq 0,
$$

and

$$
D_{t}^{\alpha} z(t)=f(t) u^{q}(t)+L(t, u(\sigma(t)))+\int_{0}^{t} h(s) u^{m}(s) \mathrm{d} s, \quad t \geq 0 .
$$

By Lemma 1 we get for any $K>0$,

$$
u(t) \leq \frac{p-1}{p} K^{\frac{1}{p}}+(k(t)+a(t) z(t)) \frac{1}{p} K^{\frac{1-p}{p}}, \quad t \geq 0 .
$$

Proceeding the similar proof of Theorem 3 in [3], we can get

$$
u(\sigma(t)) \leq \frac{p-1}{p} K^{\frac{1}{p}}+(k(t)+a(t) z(t)) \frac{1}{p} K^{\frac{1-p}{p}}, \quad t \geq 0 .
$$

From (23), (24), (25) and condition (19) we have

$$
\begin{aligned}
& D_{t}^{\alpha} z(t) \leq f(t)[k(t)+a(t) z(t)]^{\frac{q}{p}}+L\left(t, \frac{p-1}{p} K^{\frac{1}{p}}+[k(t)+a(t) z(t)] \frac{1}{p} K^{\frac{1-p}{p}}\right)+\int_{0}^{t} h(s)[k(s)+a(s) z(s)]^{\frac{m}{p}} \mathrm{~d} s \\
& \leq f(t)\left(\frac{p-q}{p} K^{\frac{q}{p}}+[k(t)+a(t) z(t)] \frac{q}{p} K^{\frac{q-p}{p}}\right)+\int_{0}^{t} h(s)\left(\frac{p-m}{p} K^{\frac{m}{p}}+[k(s)+a(s) z(s)] \frac{m}{p} K^{\frac{m-p}{p}}\right) \mathrm{d} s \\
& +L\left(t, \frac{p-1}{p} K^{\frac{1}{p}}+[k(t)+a(t) z(t)] \frac{1}{p} K^{\frac{1-p}{p}}\right)+L\left(t, \frac{p-1}{p} K^{\frac{1}{p}}+k(t) \frac{1}{p} K^{\frac{1-p}{p}}\right) \\
& -L\left(t, \frac{p-1}{p} K^{\frac{1}{p}}+k(t) \frac{1}{p} K^{\frac{1-p}{p}}\right) \\
& \leq f(t)\left(\frac{p-q}{p} K^{\frac{q}{p}}+\frac{q}{p} K^{\frac{q-p}{p}} k(t)+\frac{q}{p} K \frac{q-p}{p} a(t) z(t)\right) \\
& +\int_{0}^{t}\left(\frac{p-m}{p} K^{\frac{m}{p}} h(s)+\frac{m}{p} K^{\frac{m-p}{p}} h(s) k(s)+\frac{m}{p} K^{\frac{m-p}{p}} h(s) a(s) z(s)\right) \mathrm{d} s \\
& +M\left(t, \frac{p-1}{p} K^{\frac{1}{p}}+\frac{1}{p} K^{\frac{1-p}{p}} k(t)\right) \frac{1}{p} K^{\frac{1-p}{p}} a(t) z(t)+L\left(t, \frac{p-1}{p} K^{\frac{1}{p}}+\frac{1}{p} K^{\frac{1-p}{p}} k(t)\right) \\
& \leq f(t)\left(\frac{p-q}{p} K^{\frac{q}{p}}+\frac{q}{p} K^{\frac{q-p}{p}} k(t)\right)+\int_{0}^{t}\left(\frac{p-m}{p} K^{\frac{m}{p}} h(s)+\frac{m}{p} K^{\frac{m-p}{p}} h(s) k(s)\right) \mathrm{d} s \\
& +L\left(t, \frac{p-1}{p} K^{\frac{1}{p}}+\frac{1}{p} K^{\frac{1-p}{p}} k(t)\right)+\left[\frac{q}{p} K^{\frac{q-p}{p}} f(t) a(t)+\int_{0}^{t} \frac{m}{p} K^{\frac{m-p}{p}} h(s) a(s) \mathrm{d} s\right. \\
& \left.+M\left(t, \frac{p-1}{p} K^{\frac{1}{p}}+\frac{1}{p} K^{\frac{1-p}{p}} k(t)\right) \frac{1}{p} K^{\frac{1-p}{p}} a(t)\right] z(t) \\
& =H_{2}(t)+G_{2}(t) z(t) \text {. }
\end{aligned}
$$

By Lemma 2 we have 


$$
z(t) \leq \frac{1}{\Gamma(\alpha)} \int_{0}^{t}(t-\tau)^{\alpha-1} H_{2}(\tau) \exp \left\{-\int_{\frac{\tau^{\alpha}}{\Gamma(1+\alpha)}}^{\frac{t^{\alpha}}{\Gamma(1+\alpha)}} G_{2}\left((s \Gamma(1+\alpha))^{\frac{1}{\alpha}}\right) \mathrm{d} s\right\} \mathrm{d} \tau, \quad t \geq 0 .
$$

Combining (22) and (27), (20) can be obtained subsequently.

Theorem 3. Assume that $a(t), b(t), k(t), u(t) \in C\left(R_{+}, R_{+}\right), \alpha>0, \beta(t) \in C^{1}\left(R_{+}, R_{+}\right)$and $\beta(t)$ is nondecreasing with $\beta(t) \leq t$ for $t \geq 0$. If $u(t)$ satisfies

$$
u(t) \leq k(t)+\frac{a(t)}{\Gamma(\alpha)} \int_{0}^{t}(t-s)^{\alpha-1} \int_{0}^{\beta(s)} b(\xi) u(\xi) \mathrm{d} \xi \mathrm{d} s, \quad t \geq 0,
$$

then

$$
u(t) \leq k(t)+\frac{a(t)}{\Gamma(\alpha+1)} \int_{0}^{t}(t-\tau)^{\alpha} H_{3}(\tau) \exp \left\{-\int_{\frac{\tau^{\alpha+1}}{\Gamma(\alpha+2)}}^{\frac{t^{\alpha+1}}{\Gamma(\alpha+2)}} G_{3}\left((s \Gamma(\alpha+2))^{\frac{1}{\alpha+1}}\right) \mathrm{d} s\right\} \mathrm{d} \tau, \quad t \geq 0,
$$

where

$$
H_{3}(t)=b(\beta(t)) k(\beta(t)) \beta^{\prime}(t), \quad G_{3}(t)=a(\beta(t)) b(\beta(t)) \beta^{\prime}(t) .
$$

Proof. Let

$$
z(t)=\frac{1}{\Gamma(\alpha)} \int_{0}^{t}(t-s)^{\alpha-1} \int_{0}^{\beta(s)} b(\xi) u(\xi) \mathrm{d} \xi \mathrm{d} s
$$

then we get

$$
u(t) \leq k(t)+a(t) z(t), \quad t \geq 0 .
$$

Since $b(t), u(t)$ are both continuous functions, $\int_{0}^{\beta(s)} b(\xi) u(\xi) \mathrm{d} \xi$ is continuous and there exists a constant $M$ satisfying $\left|\int_{0}^{\beta(s)} b(\xi) u(\xi) \mathrm{d} \xi\right| \leq M$ for $t \in[0, \varepsilon]$, where $\varepsilon>0$. Then we get

$$
z(t)=\frac{1}{\Gamma(\alpha)} \int_{0}^{t}(t-s)^{\alpha-1} \int_{0}^{\beta(s)} b(\xi) u(\xi) \mathrm{d} \xi \mathrm{d} s \leq \frac{M}{\Gamma(\alpha+1)} t^{\alpha},
$$

so we get $z(0)=0$ and

$$
\begin{aligned}
D_{t}^{\alpha+1} z(t) & =b(\beta(t)) u(\beta(t)) \beta^{\prime}(t) \\
& \leq b(\beta(t))[k(\beta(t))+a(\beta(t)) z(\beta(t))] \beta^{\prime}(t) \\
& \leq b(\beta(t))[k(\beta(t))+a(\beta(t)) z(t)] \beta^{\prime}(t) \\
& =b(\beta(t)) k(\beta(t)) \beta^{\prime}(t)+a(\beta(t)) b(\beta(t)) \beta^{\prime}(t) z(t) \\
& =H_{3}(t)+G_{3}(t) z(t) .
\end{aligned}
$$

By Lemma 2 we have

$$
z(t) \leq \frac{1}{\Gamma(\alpha+1)} \int_{0}^{t}(t-\tau)^{\alpha} H_{3}(\tau) \exp \left\{-\int_{\frac{\tau^{\alpha+1}}{\Gamma(\alpha+2)}}^{\frac{t^{\alpha+1}}{\Gamma(\alpha+2)}} G_{3}\left((s \Gamma(\alpha+2))^{\frac{1}{\alpha+1}}\right) \mathrm{d} s\right\} \mathrm{d} \tau, \quad t \geq 0 .
$$

Combining (30) and (31), we get (29).

Remark 2. Considering $\beta(t)=t$ in Theorem 3, proceeding the similar proof of Theorem 3 , we can get $u(t) \leq k(t)+\frac{a(t)}{\Gamma(\alpha+1)} \int_{0}^{t}(t-\tau)^{\alpha} b(\tau) k(\tau) \exp \left\{-\int_{\frac{\tau^{\alpha+1}}{\Gamma(\alpha+2)}}^{\frac{t^{\alpha+1}}{\Gamma(\alpha+2)}}\left[a\left((s \Gamma(\alpha+2))^{\frac{1}{\alpha}}\right) b\left((s \Gamma(\alpha+2))^{\frac{1}{\alpha}}\right)\right] \mathrm{d} s\right\} \mathrm{d} \tau, \quad t \geq 0$.

Theorem 4. Assume that $a(t), k(t), u(t) \in C\left(R_{+}, R_{+}\right), \alpha>0, \beta(t) \in C^{1}\left(R_{+}, R_{+}\right)$and $\beta(t)$ is nonde- 
creasing with $\beta(t) \leq t$ for $t \geq 0, b(t, s) \in C\left(R_{+} \times R_{+}, R_{+}\right)$with $(t, s) \rightarrow \partial_{t} b(t, s) \in C\left(R_{+} \times R_{+}, R_{+}\right)$. If $u(t)$ satisfies the following form of delay integral inequality

$$
u(t) \leq k(t)+\frac{a(t)}{\Gamma(\alpha)} \int_{0}^{t}(t-s)^{\alpha-1} \int_{0}^{\beta(s)} b(s, \xi) u(\xi) \mathrm{d} \xi \mathrm{d} s, \quad t \geq 0,
$$

then

$$
u(t) \leq k(t)+\frac{a(t)}{\Gamma(\alpha+1)} \int_{0}^{t}(t-\tau)^{\alpha} H_{4}(\tau) \exp \left\{-\int_{\frac{\tau^{\alpha+1}}{\Gamma(\alpha+2)}}^{\frac{t^{\alpha+1}}{\Gamma(\alpha+2)}} G_{4}\left((s \Gamma(\alpha+2))^{\frac{1}{\alpha+1}}\right) \mathrm{d} s\right\} \mathrm{d} \tau, \quad t \geq 0,
$$

where

$$
\begin{aligned}
& H_{4}(t)=b(t, \beta(t)) k(\beta(t)) \beta^{\prime}(t)+\int_{0}^{\beta(t)} k(s) \partial_{t} b(t, s) \mathrm{d} s, \\
& G_{4}(t)=a(\beta(t)) b(t, \beta(t)) \beta^{\prime}(t)+\int_{0}^{\beta(t)} a(s) \partial_{t} b(t, s) \mathrm{d} s .
\end{aligned}
$$

Proof. Let

$$
z(t)=\frac{1}{\Gamma(\alpha)} \int_{0}^{t}(t-s)^{\alpha-1} \int_{0}^{\beta(s)} b(s, \xi) u(\xi) \mathrm{d} \xi \mathrm{d} s, \quad t \geq 0
$$

then we get

$$
u(t) \leq k(t)+a(t) z(t), \quad t \geq 0,
$$

The assumptions on $b(t), u(t)$ and $\beta(t)$ imply that $z(t)$ is nondecreasing and there exists a constant $M$ satisfying $\left|\int_{0}^{\beta(s)} b(s, \xi) u(\xi) \mathrm{d} \xi\right| \leq M$ for $t \in[0, \varepsilon]$, where $\varepsilon>0$.

Then we get

$$
z(t)=\frac{1}{\Gamma(\alpha)} \int_{0}^{t}(t-s)^{\alpha-1} \int_{0}^{\beta(s)} b(s, \xi) u(\xi) \mathrm{d} \xi \mathrm{d} s \leq \frac{M}{\Gamma(\alpha+1)} t^{\alpha}, \quad t \geq 0,
$$

so we have $z(0)=0$. For $t \geq 0$, we have

$$
D_{t}^{\alpha} z(t)=\int_{0}^{\beta(t)} b(t, s) u(s) \mathrm{d} s,
$$

and

$$
\begin{aligned}
& D_{t}^{\alpha+1} z(t) \\
& =b(t, \beta(t)) u(\beta(t)) \beta^{\prime}(t)+\int_{0}^{\beta(t)} \partial_{t} b(t, s) u(s) \mathrm{d} s \\
& \leq b(t, \beta(t))[k(\beta(t))+a(\beta(t)) z(\beta(t))] \beta^{\prime}(t)+\int_{0}^{\beta(t)} \partial_{t} b(t, s)[k(s)+a(s) z(s)] \mathrm{d} s \\
& \leq b(t, \beta(t))[k(\beta(t))+a(\beta(t)) z(t)] \beta^{\prime}(t)+\int_{0}^{\beta(t)} \partial_{t} b(t, s)[k(s)+a(s) z(t)] \mathrm{d} s \\
& =b(t, \beta(t)) k(\beta(t)) \beta^{\prime}(t)+\int_{0}^{\beta(t)} k(s) \partial_{t} b(t, s) \mathrm{d} s+\left[a(\beta(t)) b(t, \beta(t)) \beta^{\prime}(t)+\int_{0}^{\beta(t)} a(s) \partial_{t} b(t, s) \mathrm{d} s\right] z(t) \\
& =H_{4}(t)+G_{4}(t) z(t) .
\end{aligned}
$$

Using Lemma 4 to (35), we can get

$$
z(t) \leq \frac{1}{\Gamma(\alpha+1)} \int_{0}^{t}(t-\tau)^{\alpha} H_{4}(\tau) \exp \left\{-\int_{\frac{\tau^{\alpha+1}}{\Gamma(\alpha+2)}}^{\frac{t^{\alpha+1}}{\Gamma(\alpha+2)}} G_{4}\left((s \Gamma(\alpha+2))^{\frac{1}{\alpha+1}}\right) \mathrm{d} s\right\} \mathrm{d} \tau, \quad t \geq 0 .
$$

Combining (34) and (36), we get (33).

Remark 3. Considering $\partial_{t} b(t, s) \equiv 0$ and $\beta(t)=t$ in Theorem 4, we can get Remark 2 . 


\section{Applications}

In this section, we will show that the inequalities established above are useful in the research concerning the boundness, uniqueness and continuous dependence on the initial value for solutions to fractional differential equations.

\subsection{Consider the Following Fractional Differential Equation}

$$
\begin{gathered}
u^{p}(t)=u(0)+I^{\alpha} H\left(t, u(t), u(\sigma(t)), \int_{0}^{t} u^{p}(s) \mathrm{d} s\right), \quad t \geq 0, \\
u(0)=C .
\end{gathered}
$$

with the condition

$$
\begin{gathered}
u(t)=\phi(t), \quad t \in[\beta, 0], \\
|\phi(\sigma(t))| \leq|u(0)|^{\frac{1}{p}}, \quad t \geq 0, \quad \sigma(t) \leq 0,
\end{gathered}
$$

where $H(t, x, y, z) \in C\left(R_{+}^{4}, R\right), \quad p>1$ is a constant, $\sigma(t) \in C\left(R_{+}, R\right), \sigma(t) \leq t, \alpha>0$,

And

$$
-\infty<\beta=\inf \left\{\sigma(t), t \in R_{+}\right\} \leq 0, \quad \phi(t) \in C\left([\beta, 0], R_{+}\right),
$$

Example 1. Assume that $H(t, x, y, z)$ satisfies

$$
|H(t, x, y, z)| \leq g(t)|x|+f(t)|y|+|z|,
$$

where $f(t), g(t)$ are nonnegative continuous functions on $t \geq 0$, then we have the following estimate for $u(t)$,

$$
\begin{aligned}
|u(t)| \leq & \left\{|C| \exp \left[\int_{0}^{\frac{t^{\alpha}}{\Gamma(1+\alpha)}} G\left((s \Gamma(1+\alpha))^{\frac{1}{\alpha}}\right) \mathrm{d} s\right]\right. \\
& \left.+\frac{1}{\Gamma(\alpha)} \int_{0}^{t}(t-\tau)^{\alpha-1} M(\tau) \exp \left[-\int_{\frac{\tau^{\alpha}}{\Gamma(1+\alpha)}}^{\frac{t^{\alpha}}{\Gamma(+\alpha)}} G\left((s \Gamma(1+\alpha))^{\frac{1}{\alpha}}\right) \mathrm{d} s\right] \mathrm{d} \tau\right\}^{\frac{1}{p}}, \quad t \geq 0,
\end{aligned}
$$

where

$$
M(t)=\frac{p-1}{p} K^{\frac{1}{p}}(f(t)+g(t)), \quad G(t)=\frac{1}{p} K^{\frac{1-p}{p}}(f(t)+g(t))+t .
$$

Proof. By Equation (37), we have

$$
u^{p}(t)=C+\frac{1}{\Gamma(\alpha)} \int_{0}^{t}(t-s)^{\alpha-1} H\left(s, u(s), u(\sigma(s)), \int_{0}^{s} u^{p}(\xi) \mathrm{d} \xi\right) \mathrm{d} s, \quad t \geq 0 .
$$

By (39) and (41) we can get

$$
\begin{aligned}
\left|u^{p}(t)\right| & \leq|C|+\frac{1}{\Gamma(\alpha)} \int_{0}^{t}(t-s)^{\alpha-1}\left|H\left(s, u(s), u(\sigma(s)), \int_{0}^{s} u^{p}(\xi) \mathrm{d} \xi\right)\right| \mathrm{d} s \\
& \leq|C|+\frac{1}{\Gamma(\alpha)} \int_{0}^{t}(t-s)^{\alpha-1}\left[f(s)|u(\sigma(s))|+g(s)|u(s)|+\int_{0}^{s} u^{p}(\xi) \mathrm{d} \xi\right] \mathrm{d} s .
\end{aligned}
$$

With a suitable application of Theorems 1 to (42) (with $m=p, q=r=1, \quad k(t)=|C|, a(t)=1, \quad h(t)=1$ ), we can obtain the desired result. This complete the proof of Example 1.

Example 2. Assume that 


$$
\left|H\left(t, x_{1}, y_{1}, z_{1}\right)-H\left(t, x_{2}, y_{2}, z_{2}\right)\right| \leq g(t)\left|x_{1}^{p}-x_{2}^{p}\right|+f(t)\left|y_{1}^{p}-y_{2}^{p}\right|+\left|z_{1}-z_{2}\right|,
$$

where $f(t), g(t)$ are nonnegative continuous functions defined $t \geq 0, p$ is the quotient of two odd numbers. Equation (37) has a unique solution.

Proof. Suppose $u_{1}(t), u_{2}(t)$ are two solutions of Equation (37), then we have

$$
\begin{aligned}
& u_{1}^{p}(t)=C+\frac{1}{\Gamma(\alpha)} \int_{0}^{t}(t-s)^{\alpha-1} H\left(s, u_{1}(s), u_{1}(\sigma(s)), \int_{0}^{s} u_{1}^{p}(\xi) \mathrm{d} \xi\right) \mathrm{d} s, \quad t \geq 0, \\
& u_{2}^{p}(t)=C+\frac{1}{\Gamma(\alpha)} \int_{0}^{t}(t-s)^{\alpha-1} H\left(s, u_{2}(s), u_{2}(\sigma(s)), \int_{0}^{s} u_{2}^{p}(\xi) \mathrm{d} \xi\right) \mathrm{d} s, \quad t \geq 0 .
\end{aligned}
$$

Furthermore,

$u_{1}^{p}(t)-u_{2}^{p}(t)=\frac{1}{\Gamma(\alpha)} \int_{0}^{t}(t-s)^{\alpha-1}\left[H\left(s, u_{1}(s), u_{1}(\sigma(s)), \int_{0}^{s} u_{1}^{p}(\xi) \mathrm{d} \xi\right)-H\left(s, u_{2}(s), u_{2}(\sigma(s)), \int_{0}^{s} u_{2}^{p}(\xi) \mathrm{d} \xi\right)\right] \mathrm{d} s$

which implies

$$
\begin{aligned}
& \left|u_{1}^{p}(t)-u_{2}^{p}(t)\right| \\
& \quad \leq \frac{1}{\Gamma(\alpha)} \int_{0}^{t}(t-s)^{\alpha-1}\left[g(s)\left|u_{1}^{p}(s)-u_{2}^{p}(s)\right|+f(s)\left|u_{1}^{p}(\sigma(s))-u_{2}^{p}(\sigma(s))\right|+\int_{0}^{s}\left|u_{1}^{p}(\xi)-u_{2}^{p}(\xi)\right| \mathrm{d} \xi\right] \mathrm{d} s,
\end{aligned}
$$

Through a suitable application of Theorem 1 to (44) (with $p=q=r=m=1, k(t)=0, a(t)=h(t)=1$ ), we can obtain

$$
\left|u_{1}^{p}(t)-u_{2}^{p}(t)\right| \leq 0,
$$

which implies $u_{1}(t) \equiv u_{2}(t)$. So Equation (37) has a unique solution.

Example 3. Suppose that $u(t)$ is the solution of (37) and $\tilde{u}(t)$ be the solution of the following fractional integral equation,

$$
\begin{gathered}
\tilde{u}^{p}(t)=\tilde{u}(0)+I^{\alpha} H\left(f(t, \tilde{u}(t), \tilde{u}(\sigma(t))), \int_{0}^{t} \tilde{u}^{p}(s) \mathrm{d} s\right), \quad t \geq 0, \\
\tilde{u}(0)=\tilde{C} .
\end{gathered}
$$

If $H(t, x, y, z)$ satisfies the condition (43), then the solution of Equation (37) depends on the initial value $C$ continuously.

Proof. By Equation (45), we have

$$
\tilde{u}^{p}(t)=\tilde{C}+\frac{1}{\Gamma(\alpha)} \int_{0}^{t}(t-s)^{\alpha-1} H\left(s, \tilde{u}(s), \tilde{u}(\sigma(s)), \int_{0}^{s} \tilde{u}^{p}(\xi) \mathrm{d} \xi\right) \mathrm{d} s, \quad t \geq 0,
$$

so we get

$$
\begin{aligned}
& u^{p}(t)-\tilde{u}^{p}(t) \\
& =C-\tilde{C}+\frac{1}{\Gamma(\alpha)} \int_{0}^{t}(t-s)^{\alpha-1}\left[H\left(s, u(s), u(\sigma(s)), \int_{0}^{s} u^{p}(\xi) \mathrm{d} \xi\right)-H\left(s, \tilde{u}(s), \tilde{u}(\sigma(s)), \int_{0}^{s} \tilde{u}^{p}(\xi) \mathrm{d} \xi\right)\right] \mathrm{d} s .
\end{aligned}
$$

Furthermore

$$
\begin{aligned}
& \left|u^{p}(t)-\tilde{u}^{p}(t)\right| \\
& \quad \leq|C-\tilde{C}|+\frac{1}{\Gamma(\alpha)} \int_{0}^{t}(t-s)^{\alpha-1}\left[g(s)\left|u^{p}(s)-\tilde{u}^{p}(s)\right|+f(s)\left|u^{p}(\sigma(s))-\tilde{u}^{p}(\sigma(s))\right|+\int_{0}^{s}\left|u^{p}(\xi)-\tilde{u}^{p}(\xi)\right| \mathrm{d} \xi\right] \mathrm{d} s .
\end{aligned}
$$

Apply Theorem 1 to (47) (with $p=q=r=m=1, \quad h(t)=1, \quad k(t)=|C-\tilde{C}|, \quad a(t)=1$ ), we get 


$$
\left|u^{p}(t)-\tilde{u}^{p}(t)\right| \leq|C-\tilde{C}| \exp \left[\int_{0}^{\frac{t^{\alpha}}{\Gamma(1+\alpha)}} G\left((s \Gamma(1+\alpha))^{\frac{1}{\alpha}}\right) \mathrm{d} s\right],
$$

where $G(t)=f(t)+g(t)+t$. This gives that the solutions of Equation (37) depends on the initial value $C$ continuously.

\subsection{Consider the Following Fractional Differential Equation}

$$
\begin{gathered}
u(t)=u(0)+I^{0.5} \int_{0}^{0.5} s u(s) \mathrm{d} s, \quad t \geq 0, \\
u(0)=C .
\end{gathered}
$$

Example 4. Assume that $u(t)$ is a solution of Equation (48), then $u(t)$ is bounded.

Proof. By Equation (48) we can get

$$
u(t)=C+\frac{1}{\Gamma(0.5)} \int_{0}^{t}(t-s)^{-0.5} \int_{0}^{s^{0.5}} \xi u(\xi) \mathrm{d} \xi \mathrm{d} s, \quad t \geq 0 .
$$

with a suitable application of Theorem 3 to (49) (with $k(t)=C, a(t)=1, \alpha=0.5, b(t)=t$, $\beta(t)=t^{0.5} \leq t$ for $\left.t \geq 0, H_{3}(t)=\frac{C}{2}, G_{3}(t)=\frac{1}{2}\right)$, we have

$$
\begin{aligned}
u(t) & \leq C+\frac{C}{2 \Gamma(1.5)} \int_{0}^{t}(t-\tau)^{0.5} \exp \left\{-\int_{\frac{\tau^{2.5}}{2 \Gamma(2.5)}}^{\frac{t^{1.5}}{2 \Gamma(2 .)}} \frac{1}{2} \mathrm{~d} s\right\} \mathrm{d} \tau \\
& \leq C+\frac{C}{2 \Gamma(1.5)} \int_{0}^{t}(t-\tau)^{0.5} \mathrm{~d} \tau \\
& =C+\frac{C}{2 \Gamma(1.5)} B(1,1.5) t^{1.5} \\
& =C\left[1+\frac{t^{1.5}}{2 \Gamma(2.5)}\right],
\end{aligned}
$$

where we used $\exp \left\{-\int_{\frac{t^{1.5}}{2 \Gamma(2.5)}}^{\frac{t^{1.5}}{2 \Gamma(2.5)}} \frac{1}{2} \mathrm{~d} s\right\} \leq 1$. This complete the proof of Example 4 .

Example 5. If $u(t)$ is a solution of (48), then it has a unique solution.

Proof. Suppose $u_{1}(t), u_{2}(t)$ are two solutions of Equation (48), then we have

$$
\begin{aligned}
& u_{1}(t)=C+\frac{1}{\Gamma(0.5)} \int_{0}^{t}(t-s)^{-0.5} \int_{0}^{s^{0.5}} \xi u_{1}(\xi) \mathrm{d} \xi \mathrm{d} s, \quad t \geq 0 \\
& u_{2}(t)=C+\frac{1}{\Gamma(0.5)} \int_{0}^{t}(t-s)^{-0.5} \int_{0}^{s^{0.5}} \xi u_{2}(\xi) \mathrm{d} \xi \mathrm{d} s, \quad t \geq 0
\end{aligned}
$$

Furthermore,

$$
u_{1}(t)-u_{2}(t)=\frac{1}{\Gamma(0.5)} \int_{0}^{t}(t-s)^{-0.5} \int_{0}^{s^{0.5}} \xi\left[u_{1}(\xi)-u_{2}(\xi)\right] \mathrm{d} \xi \mathrm{d} s
$$

which implies

$$
\left|u_{1}(t)-u_{2}(t)\right| \leq \frac{1}{\Gamma(0.5)} \int_{0}^{t}(t-s)^{-0.5} \int_{0}^{s^{0.5}} \xi\left|u_{1}(\xi)-u_{2}(\xi)\right| \mathrm{d} \xi \mathrm{d} s .
$$

With a suitable application of 3 to (50) (with $u(t)=\left|u_{1}(t)-u_{2}(t)\right|, k(t)=0, a(t)=1, \quad \alpha=0.5, \quad b(t)=t$, 
$\beta(t)=t^{0.5} \leq t$ for $\left.t \geq 0\right)$, we can obtain

$$
\left|u_{1}(t)-u_{2}(t)\right| \leq 0,
$$

which implies $u_{1}(t) \equiv u_{2}(t)$. So Equation (48) has a unique solution.

Example 6. Suppose that $u(t)$ is the solution of (48) and $\tilde{u}(t)$ is the solution of the following fractional integral equation,

$$
\begin{gathered}
\tilde{u}(t)=\tilde{u}(0)+I^{0.5} \int_{0}^{0.5} s \tilde{u}(s) \mathrm{d} s, \quad t \geq 0, \\
\tilde{u}(0)=\tilde{C} .
\end{gathered}
$$

Then all the solutions of Equation (48) depend on the initial value $C$ continuously.

Proof. By Equation (51), we have

$$
\tilde{u}(t)=\tilde{C}+\frac{1}{\Gamma(0.5)} \int_{0}^{t}(t-s)^{-0.5} \int_{0}^{s^{0.5}} \xi \tilde{u}(\xi) \mathrm{d} \xi \mathrm{d} s, \quad t \geq 0,
$$

so we get

$$
u(t)-\tilde{u}(t)=C-\tilde{C}+\frac{1}{\Gamma(0.5)} \int_{0}^{t}(t-s)^{-0.5} \int_{0}^{s^{0.5}} \xi[u(\xi)-\tilde{u}(\xi)] \mathrm{d} \xi \mathrm{d} s, \quad t \geq 0
$$

Furthermore

$$
|u(t)-\tilde{u}(t)|=|C-\tilde{C}|+\frac{1}{\Gamma(0.5)} \int_{0}^{t}(t-s)^{-0.5} \int_{0}^{s^{0.5}} \xi|u(\xi)-\tilde{u}(\xi)| \mathrm{d} \xi \mathrm{d} s, \quad t \geq 0 .
$$

Apply Theorem 3 to (52) (with $k(t)=|C-\tilde{C}|, a(t)=1, \quad b(t)=t, \alpha=0.5, \beta(t)=t^{0.5} \leq t$ for $t \geq 0$, $\left.H_{4}(t)=\frac{|C-\tilde{C}|}{2}, G_{4}(t)=\frac{1}{2}\right)$, we get

$$
\begin{aligned}
|u(t)-\tilde{u}(t)| & \leq|C-\tilde{C}|+\frac{|C-\tilde{C}|}{2 \Gamma(1.5)} \int_{0}^{t}(t-\tau)^{-0.5} \exp \left\{-\int_{\frac{\tau^{\prime} .5}{\Gamma(2.5)}}^{\frac{t^{1.5}}{\Gamma(2.5}} \frac{1}{2} \mathrm{~d} s\right\} \mathrm{d} \tau \\
& \leq|C-\tilde{C}|\left[1+\frac{1}{2 \Gamma(1.5)} \int_{0}^{t}(t-\tau)^{0.5} d \tau\right] \\
& =|C-\tilde{C}|\left[1+\frac{1}{2 \Gamma(1.5)} B(1,1.5) t^{1.5}\right] \\
& =|C-\tilde{C}|\left[1+\frac{t^{1.5}}{2 \Gamma(2.5)}\right],
\end{aligned}
$$

where we use the fact that $\exp \left\{-\int_{\frac{\tau^{1.5}}{\Gamma(2.5)}}^{\frac{t^{1.5}}{\Gamma(2.5)}} \frac{1}{2} \mathrm{~d} s\right\} \leq 1$

This gives that $u(t)$ depends on the initial value $C$ continuously.

\section{Acknowledgements}

We thank the Editor and the referee for their comments. This work is supported by National Science Foundation of China (11171178 and 11271225).

\section{References}

[1] Jiang, F.C. and Meng, F.W. (2007) Explicit Bounds on Some New Nonlinear Integral Inequalities with Delay. Journal 
of Computational and Applied Mathematics, 205, 479-486. http://dx.doi.org/10.1016/j.cam.2006.05.038

[2] Zhang, H.X. and Meng, F.W. (2008) Integral Inequalities in Two Independent Variables for Retarded Volterra Equations. Applied Mathematics and Computation, 199, 90-98. http://dx.doi.org/10.1016/j.amc.2007.09.026

[3] Yuan, Z.L., Yuan, X.W., Meng, F.W. and Zhang, H.X. (2008) Some New Delay Integral Inequalities and Their Applications. Journal of Computational and Applied Mathematics, 180, 191-200.

[4] Zheng, B. (2013) Some New Gronwall-Bellman-Type Inequalities Based on the Modified Riemann-Liouville Fractional Derivative. Hindawi Publishing Corporation Journal of Applied Mathematics, 2013, Article ID: 341706. http://dx.doi.org/10.1155/2013/341706

[5] Zheng, B. (2014) Explicit Bounds Derived by Some New Inequalities and Applications in Fractional Integral Equations. Journal of Inequalities and Applications, 2014, 4.

http://www.journalofinequalitiesandapplications.com/content/2014/1/4 http://dx.doi.org/10.1186/1029-242X-2014-4

[6] Zheng, B. and Feng, Q.H. (2013) New Gronwall-Bellman Type Inequalities and Applications in the Analysis for Solutions to Fractional Differential Equations. Hindawi Publishing Corporation Abstract and Applied Analysis, 2013, Article ID: 705126. http://dx.doi.org/10.1155/2013/705126

[7] Denton, Z. and Vatsala, A.S. (2010) Fractional Integral Inequalities and Applications. Computers and Mathematics with Applications, 59, 1087-1094. http://dx.doi.org/10.1016/j.camwa.2009.05.012

[8] Ye, H.P., Gao, J.M. and Ding, Y.S. (2007) A Generalized Gronwall Inequality and Its Application to a Fractional Differential Equation. Journal of Mathematical Analysis and Applications, 328, 1075-1081. http://dx.doi.org/10.1016/j.jmaa.2006.05.061

[9] Khalil, R., Al Horani, M., Yousef, A. and Sababheh, M. (2014) A New Definition of Fractional Derivative. Journal of Computational and Applied Mathematics, 264, 65-70. http://dx.doi.org/10.1016/j.cam.2014.01.002

[10] Jessada, T., Ntouyas, S.K. and Weerawat, S. (2014) Some New Riemann-Liouville Fractional Integral Inequalities. Hindawi Publishing Corporation International Journal of Mathematics and Mathematical Sciences, 2014, Article ID: 869434. http://dx.doi.org/10.1155/2014/869434

[11] Jalilian, Y. and Jalilian, R. (2013) Existence of Solution for Delay Fractional Differential Equations. Mediterranean Journal of Mathematics, 10, 1731-1747. 